\title{
PRODUCTS OF ABELIAN HOPFIAN GROUPS
}

\author{
GILBERT BAUMSLAG 1
}

(Received 24 October 1966)

\section{Introduction}

1.1 Let $\mathfrak{N}_{k}$ be the variety of all nilpotent groups of class at most $k$.

The purpose of this note is to prove the following

THEOREM 1. Let $\mathfrak{B}$ be a variety of groups containing $\mathfrak{R}_{2}$, let $A$ and $B$ be torsion-free abelian hopfian groups and let $P$ be the free $\mathfrak{B}$-product ${ }^{2}$ of $A$ and $B$. If $P$ is residually torsion-free nilpotent, then $P$ is hopfian.

Let $\widetilde{S}_{k}$ denote the variety of all soluble groups of derived length at most $k$. Since the free $\varsigma_{k}$-product of torsion-free abelian groups is residually torsion-free nilpotent ([2]) Theorem 1 applies and we have, therefore, the

COROLLARY $1^{3}$. For every $k \geqq 2$ the free $\mathfrak{S}_{k}$-product of any pair of torsionfree abelian hopfian groups is hopfian.

Corollary 1 is somewhat surprisingly, no longer valid when $k=1$; in this case $P$ is the direct product of $A$ and $B$, and A.L.S. Corner [3] has constructed a number of extraordinary counter-examples.

1.2 The proof of Theorem 1 is not difficult, depending essentially on an analysis of the centralizers of certain elements in the free $\mathfrak{R}_{2}$-product of torsion-free abelian groups.

\section{A free $\mathfrak{R}_{2}$-product}

2.1 Let $S$ be the set of all quintuplets of rational numbers $(r, s, t, u, v)$. We turn $S$ into a group by defining

$(r, s, t, u, v) \cdot\left(r^{*}, s^{*}, t^{*}, u^{*}, v^{*}\right)=\left(r+r^{*}, s+s^{*}, t+t^{*}, u+u^{*}-s r^{*}, v+v^{*}-t r^{*}\right)$.

The set of elements of the form

$$
(r, 0,0,0,0)
$$

1 The author thanks the Sloan Foundation and the National Science Foundation for their support.

2 I.e. the free product $F$ of $A$ and $B$ modulo the verbal subgroup of $F$ determined by $\mathfrak{B}$ (cf. S. Moran [7]).

3 This is a special case of a theorem announced in [1]. 
is a subgroup $C$ of $S$ isomorphic to the additive group $Q$ of rational numbers. Similarly the set of elements of the form

$$
(0, s, t, 0,0)
$$

is a subgroup $D$ isomorphic to $Q \times Q$. (Indeed $S$ is actually the free $\mathfrak{R}_{2}$ product of $C$ and $D$.) $S$ is readily seen to be nilpotent of class two.

\section{Centralizers}

3.1 Let $A$ and $B$ be torsion-free abelian groups and let $P$ be their free $\mathfrak{R}_{2}$-product. We shall prove here that if $a \in A(a \neq 1), b \in B(b \neq 1)$, $z \in Z$, the centre of $P$, then the centralizer $C(a b z)$ of $a b z$ is locally cyclic modulo $Z$.

3.2 We begin with the following simple

Lemma 1. If $b^{\prime}\left(b^{\prime} \in B\right)$ centralizes abz, then $b^{\prime}=1$.

Proof. Consider the group $S$ of 2.1. Now assume $b^{\prime} \neq 1$. By a characteristic property of divisible groups the mappings

$$
a \rightarrow(1,0,0,0,0), \quad b^{\prime} \rightarrow(0,1,0,0,0)
$$

can be continued to homomorphisms of $A$ into $C$ and $B$ into $D$, respectively, and hence to a homomorphism $\eta$ of $P$ into $S$.

But, remembering $S$ is nilpotent of class two ${ }^{4}$,

$$
\left[(a b z) \eta, b^{\prime} \eta\right]=\left[a \eta, b^{\prime} \eta\right]=(0,0,0,1,0) \neq 1 .
$$

This completes the proof of Lemma 1.

Corollary 1. If $b^{\prime}$ centralizes $a, b^{\prime}=1$.

3.3 Next we need

LEMMA 2. If $b^{\prime}$ and $b$ generate a free abelian subgroup of $B$ of rank two, then $a^{\prime} b^{\prime} z^{\prime}$ does not centralize abz for any choice of $a^{\prime} \in A, z^{\prime} \in Z$.

Proof. Consider again the group $S$. Choose a homomorphism $\eta$ of $P$ into $S$ as in the proof of Lemma 1, which takes

$$
a \text { to }(1,0,0,0,0), b \text { to }(0,1,0,0,0), b^{\prime} \text { to }(0,0,1,0,0) \text {. }
$$

Then, writing $a^{\prime}=a^{r}$ where $r$ may be rational (and therefore interpreted in the obvious way), $[x, y]$.

1f $x, y$ are elements of a group we denote the commutator $x^{-1} y^{-1} x y$ of $x$ and $y$ by 


$$
\begin{aligned}
& {\left[(a b z) \eta,\left(a^{\prime} b^{\prime} z^{\prime}\right) \eta\right]=\left[a \eta, b^{\prime} \eta\right]\left[b \eta, s^{\prime} \eta\right]} \\
& =[(1,0,0,0,0),(0,0,1,0,0)]\left[(0,1,0,0,0),(1,0,0,0,0)^{r}\right] \\
& =(0,0,0,1,0)(0,0,0,0, r) \\
& =(0,0,0,1, r) .
\end{aligned}
$$

This proves Lemma 2 .

3.4 Finally we arrive at the promised

Proposition 1. $\quad C(a b z) / Z$ is locally cyclic.

Proof. Let $a^{\prime} b^{\prime} z^{\prime} \in C(a b z)$. By Lemma 1 (and its analogue for $a^{\prime}$ ) either $a^{\prime} b^{\prime} z^{\prime} \in Z$ or else

$$
a^{\prime} \neq 1, \quad b^{\prime} \neq 1 .
$$

But by Lemma 2 (and its analogue for the subgroup generated by $a$ and $a^{\prime}$ )

$$
g p\left(a, a^{\prime}\right) \text { is cyclic, } g p\left(b, b^{\prime}\right) \text { is cyclic. }
$$

We need only one of these remarks. Thus choose integers $r$ and $s$ so that

Then, obviously

$$
a^{r}\left(a^{\prime}\right)^{s}=1 \quad(r \neq 0 \neq s) .
$$

$$
(a b z)^{r}\left(a^{\prime} b^{\prime} z^{\prime}\right)^{s} \in C(a b z) .
$$

But Lemma 1 applies and so we find $(a b z)^{r}\left(a^{\prime} b^{\prime} z^{\prime}\right)^{s} \in Z$; in other words $a b z$ and $a^{\prime} b^{\prime} z^{\prime}$ generate a cyclic subgroup modulo $Z$ and so the proof of the proposition is complete.

\section{The proof of Theorem 1}

4.1 Let $P$ be the free $\mathfrak{B}$-product $\left(\mathfrak{B} \supseteqq \mathfrak{R}_{2}\right)$ of the torsion-free abelian hopfian groups $A$ and $B$ and let $\eta$ be a homomorphism of $P$ onto $P$. Let

$$
P=P_{1} \geqq P_{2} \geqq P_{3} \geqq \cdots
$$

be the lower central series of $P$. Since $\mathfrak{B}$ contains $\mathfrak{R}_{2}, P / P_{3}$ is the free $\Re_{2}$-product of $A$ and $B$. Notice that the centre of $P / P_{3}$ is simply $P_{2} / P_{3}$.

We consider first the case when both $A \eta$ and $B \eta$ are not locally cyclic modulo $P_{2}$. Look at $A \eta$. Since $A \eta P_{2} / P_{2}$ is not locally cyclic, $A \eta$ does not contain an element of the form $a b z\left(a \neq 1, b \neq 1, a \in A, b \in B, z \in P_{2}\right)$ (Proposition 1). So, by Corollary 1, either

Similarly

$$
A \eta \leqq A P_{2} \text { or } A \eta \leqq B P_{2} \text {. }
$$

$$
B \eta \leqq B P_{2} \text { or } B \eta \leqq A P_{2} .
$$

As suggested by the order, we claim therefore that either $A \eta \leqq A P_{2}$ and 
$B \eta \leqq B P_{2}$ or $A \eta \leqq B P_{2}$ and $B \eta \leqq A P_{2}$ since $P$ is certainly not generated by $A$ modulo $P_{2}$. But, in the event that the latter possibility is in force, we may equally concern ourselves with $\eta^{2}$ where

$$
A \eta^{2} \leqq A P_{2} \text { and } B \eta^{2} \leqq B P_{2} \text {. }
$$

Now this means that, modulo $P_{2}, \eta$ maps $A$ isomorphically onto $A$ and $B$ isomorphically onto $B$. Since $P$ is the free $B$-product of $A$ and $B$, every pair of automorphisms of $A$ and $B$ respectively can simultaneously be extended to an automorphism of $P$. Thus we may assume that $\eta$ induces the identity homomorphism of $P$ modulo $P_{2}$. But then $\eta$ is certainly monomorphic (P. Hall [5], Lemma 1).

4.2 We consider next the case where $A \eta P_{2} / P_{2}$ is not locally cyclic, but $B \eta P_{2} / P_{2}$ is locally cyclic. It then follows from the argument of $\mathbf{4 . 1}$ that we may assume that $\eta$ leaves $A$ fixed modulo $P_{2}$ and also that $\eta$ leaves $B$ fixed modulo $A P_{2}$.

Now embed $A$ and $B$ in minimal torsion-free divisible groups $\bar{A}$ and $\bar{B}$ respectively (see, for example, L. Fuchs [4]). Let $R$ be the free $\mathfrak{B}$-product of $\bar{A}$ and $\bar{B}$. It is not difficult to prove that $A$ and $B$ generate in $R$ their free $\mathfrak{B}$-product (isomorphic to) $P$ and that $R$ is residually torsion-free nilpotent (see, for example, the argument on pages 364 and 365 of [2]). Now for each $i$ let

$$
R(i)=R / T_{i},
$$

where $T_{i}$ is the inverse image of the torsion-subgroup of $R / R_{i}$. As $R$ is residually torsion-free nilpotent, the $T_{i}$ have intersection 1 . Notice that $R(i)$ is a torsion-free nilpotent divisible group since it is Cernikov complete (see, for example, A. G. Kurosh [6], vol. 2, p. 233). Consider now

$$
A \eta T_{i} / T_{i} \text { and } B \eta T_{i} / T_{i} \text {. }
$$

Since $R(i)$ is a torsion-free divisible nilpotent group, both $A \eta T_{i} \mid t_{i}$ and $B \eta T_{i} / T_{i}$ can be enlarged to minimal torsion-free divisible abelian groups (see, for example, A. G. Kurosh [6], vol. 2, p. 256). So by a characteristic property of divisible groups the mappings which are induced by $\eta$ in $A T_{i} / T_{i}$ and $B T_{i} / T_{i}$ can be extended to homomorphisms $\alpha$ and $\beta$ say from $\bar{A} T_{i} / T_{i}$ and $B T_{i} / T_{i}$ respectively into $R(i)$. But $R(i)$ is clearly the "freest" group in $\mathfrak{B}$ which is torsion-free and nilpotent of class at most $i-1$, generated by (copies of) $\bar{A}$ and $\bar{B}$. So $\alpha$ and $\beta$ can be simultaneously extended to a homomorphism $\gamma$ of $R(i)$ into $R(i)$. But $\gamma$ leaves $\bar{A} T_{i} / T_{i}$ fixed modulo the derived group of $R(i)$ and also $B T_{i} / T_{i}$ fixed modulo the group generated by $\bar{A} T_{i} / T_{i}$ and the derived group of $R(i)$. Thus the images of $\bar{A} T_{i} / T_{i}$ and $B T_{i} / T_{i}$ under $\gamma$ generate $R(i)$ modulo its derived group. Hence, remembering $R(i)$ is nilpotent, $\left(A T_{i} / T_{i}\right) \gamma$ and $\left(B T_{i} / T_{i}\right) \gamma$ generate $R(i)$ and so $\gamma$ 
is onto. Now pick an element $b \neq 1, b \in \bar{B} T_{i} / T_{i}$. Then $b \gamma=b a$ modulo the derived group of $R(i)$, where $a \in \bar{A} T_{i} / T_{i}$. Embed $b a^{-1}$ in a copy of the rationals inside $R(i)$. Then the mapping $\delta_{2}$ defined by

$$
b \delta_{2}=b a^{-1}
$$

can be continued to a homomorphism of $\bar{B} T_{i} / T_{i}$ into $R(i)$. Since $\bar{B} T_{i} / T_{i}$ is in fact isomorphic to the additive group of rationals, $\delta_{2} \gamma$ leaves $B T_{i} / T_{i}$ fixed modulo the derived group of $R(i)$. Now let $\delta_{1}$ be the restriction of $\gamma$ to $\bar{A} T_{i} / T_{i}$ and extend $\delta_{1}$ and $\delta_{2}$ to a homomorphism $\delta$ of $R(i)$ into $R(i)$. Now $\delta$ is onto as before and $\gamma \delta$ leaves $R(i)$ fixed modulo its derived group. So $\gamma \delta$ is an automorphism by Lemma 1 of $P$. Hall [5]. So $\gamma$ is one-to-one and hence $\eta$ must be one-to-one as the $T_{i}$ intersect trivially. This completes the second part of the analysis.

4.3 Suppose finally that $A \eta$ and $B \eta$ are both locally cyclic modulo $P_{2}$. It follows that both $A$ and $B$ are locally cyclic. Hence $P$ is itself residually a poly-locally-infinite-cyclic group and therefore clearly hopfian.

The completes the proof of the theorem.

\section{References}

[1] G. Baumslag, 'Hopficity and abelian groups', Topics in abelian groups, Proceedings of symposium on abelian groups, New Mexico State University (1962), 331-335.

[2] G. Baumslag, 'On the residual nilpotence of some varietal products', Trans. American Math. Soc. 109 (1963), 357-365.

[3] A. L. S. Corner, 'Three examples on hopficity in torsion-free abelian groups', Acta Math. Acad. Sci. Hungar. 16 (1965), 303-310.

[4] L. Fuchs, Abelian groups (Pergamon, Budapest, 1958).

[5] P. Hall, 'The splitting properties of relatively free groups', Proc. London Math. Soc. 4 (1954), 343-356.

[6] A. G. Kurosh, The theory of groups Volume 2. (Chelsea, New York 1955).

[7] S. Moran, 'Associative operations on groups. I', Proc. London Math. Soc. 6 (1956), 581-596.

Graduate Center

The City University of New York 